\title{
Anestesia em Paciente com Angioedema Hereditário
}

\author{
Anesthesia in a Patient with Hereditary Angioedema
}

\author{
Daniela Nunes Oliveira ${ }^{1}$, Anita Perpetua Carvalho Rocha de Castro ${ }^{1}$, Catharina \\ Borges de Oliveira ${ }^{1}$, Felipe Augusto Ribeiro Valadares ${ }^{1}$, Zenon Rei de Brito Borges ${ }^{1}$ \\ ${ }^{I}$ Serviço de Anestesiologia do Hospital Santa Izabel; Salvador, Bahia, Brasil
}

Correspondence addresses: Dra. Daniela Nunes Oliveira danih.oliveira@hotmail.com

Received: April 12, 2019

Revised: May 31, 2019

Accepted: June 16, 2019

Published: June 28, 2019

Data Availability Statement: All relevant data are within the paper and its Supporting Information files.

Funding: This work was the result of authors' initiative. There was no support of research or publication funds.

Competing interests: The authors have declared that no competing interests exist.

Copyright

(C) 2019 by Santa Casa

de Misericórdia da Bahia.

All rights reserved.

ISSN: 2526-5563
Introdução: $O$ angioedema hereditário (AEH) é uma doença genética autossômica dominante caracterizada por deficiência da enzima inibidora de C1-esterase (C1-INH). Na via inflamatória, a C1-INH controla a produção de bradicinina, regulando a vasodilatação e aumento da permeabilidade vascular. Desta forma, os pacientes podem apresentar sintomas de angioedema não responsivo a antihistamínicos, corticoides e epinefrina. Relato de Caso: Paciente do sexo feminino, portadora de AEH, admitida para realização de Endoscopia Digestiva Alta (EDA). Em uso de ácido tranexâmico e anticoncepcional oral com progesterona. Solicitadas, em avaliação pré-anestésica, reserva de vaga em unidade fechada e plasma fresco congelado. Submetida a EDA sob anestesia geral venosa - sem intercorrências. Após o procedimento paciente apresentou edema em mãos e face, sendo administrado ácido tranexâmico $500 \mathrm{mg}$, com melhora gradual do quadro. Paciente mantida em observação em unidade aberta sem novas crises, recebendo alta após 24 horas. Discussão: O AEH é uma patologia genética rara e, por isto, pouco conhecida pelos profissionais de saúde. Os pacientes podem apresentar quadro de angioedema na presença de fatores desencadeantes como estresse, alterações hormonais e procedimentos cirúrgicos. No contexto da anestesiologia, o conhecimento da patologia tem importância no manejo de possíveis complicações, como edema de vias aéreas. $O$ manejo do quadro agudo é feito com reposição do concentrado de C1-INH, antagonistas do receptor de bradicinina, inibidores da calicreína ouplasma fresco e, em casos leves, ácido tranexâmico. Identificação precoce de possíveis complicações e instituição de terapêutica adequada são essenciais para garantir melhor prognóstico para estes pacientes.

Palavras-chave: Angioedema Hereditário; Bradicinina; Anestesiologia.

Introduction: Hereditary angioedema (HAE) is an autosomal dominant genetic disorder characterized by deficiency of the $\mathrm{C} 1$-esterase inhibitory enzyme (C1$\mathrm{INH}$ ). In the inflammatory pathway, C1-INH controls the production of bradykinin, regulating vasodilation and increased vascular permeability. So, patients may present symptoms of angioedema not responsive to antihistamines, corticosteroids and epinephrine. Case Report: A female-patient with HAE, using tranexamic acid and oral contraceptive with progesterone, was admitted for upper gastrointestinal endoscopy. After the preanesthetic evaluation, it was requested reservation in the intensive care unit and fresh-frozen plasma. The patient was submitted to endoscopy under general venous anesthesia without intercurrences. After the procedure, the patient presented hand and face edema and tranexamic acid 500mg was administered, with gradual improvement of the condition. The patient remained in open-unit observation without new complications and received discharge after 24 hours. Discussion: HAE is a rare genetic disorder and, therefore, poorly understood by health professionals. 
Patients may present angioedema in the presence of triggering factors such as stress, hormonal changes and surgical procedures. In the context of anesthesiology, knowledge of pathology is important in the management of possible complications, such as airway edema. The acute condition is treated with replacement of the C1-INH concentrate, bradykinin receptor antagonists, kallikrein inhibitors or fresh-frozen plasma and, in mild cases, tranexamic acid. Early identification of possible complications and establishment of appropriate therapy are essential to ensure better prognoses for these patients.

Keywords: Hereditary Angioedema; Bradykinin; Anesthesiology.

\section{Introdução}

$\mathrm{O}$ angioedema hereditário (AEH) é uma doença genética autossômica dominante com prevalência aproximada de 1:50.000, mais comum em mulheres e caracterizada por deficiência quantitativa e/ou qualitativa da enzima inibidora de $\mathrm{C} 1$-esterase $\left(\mathrm{C} 1\right.$-INH). ${ }^{1}$

$\mathrm{Na}$ via inflamatória, a C1-INH controla a produção de bradicinina mediada por calicreína, regulando a vasodilatação $\mathrm{e}$ aumento da permeabilidade vascular. Desta forma, pacientes com deficiência da $\mathrm{C} 1$ $\mathrm{INH}$, na presença de fatores desencadeantes, tem aumento do nível sérico da bradicinina e podem apresentar angioedema não responsivo às medidas habitualmente utilizadas, como anti-histamínicos, corticoides e epinefrina., ${ }^{2,3}$

Oconhecimento acercadoAEHéimportante, pois, apesar de ser uma patologia rara, as complicações no período perioperatório, se não reconhecidas e tratadas adequadamente, aumentam bastante a morbi-mortalidade destes pacientes.

\section{Relato de Caso}

Paciente C.M.L.A., sexo feminino, 34 anos, $78 \mathrm{~kg}$, com diagnóstico de angioedema hereditário com inibidor de $\mathrm{C} 1$ esterase normal e passado de asma na infância. Admitida em serviço de gastroenterologia hospitalar para realização de Endoscopia Digestiva Alta para investigação de dor abdominal e azia. Em uso de ácido tranexâmico $250 \mathrm{mg}$ a cada 6 horas (iniciado uma semana antes do procedimento e orientado por médico assistente manter por 3 dias após) e anticoncepcional oral apenas com progesterona para tratamento da patologia de base. Exame físico, laboratoriais, radiografia de tórax e ecocardiograma sem anormalidades. Solicitado pela equipe de anestesiologia em avaliação pré-anestésica reserva de vaga em unidade fechada (UTI) e de plasma fresco congelado.

Submetida a Endoscopia Digestiva Alta sob anestesia geral venosa. Feita monitorização multiparamétrica e venóclise. Administrado propofol $1 \% 3 \mathrm{mg} / \mathrm{Kg}$ associado a lidocaína $2 \%$ sem vasoconstrictor $1 \mathrm{mg} / \mathrm{Kg}$ por via endovenosa. Paciente mantida em ventilação espontânea com suporte de oxigênio sob cateter nasal $2 \mathrm{~L} / \mathrm{min}$. Procedimento realizado sem intercorrências e com duração de 15 minutos. Após 1 hora e 15 minutos do término do procedimento a paciente apresentou edema em mãos e em face à esquerda (Figuras 1 e 2), sendo administrado ácido tranexâmico $500 \mathrm{mg}$, com melhora gradual do quadro. Paciente mantida em observação em unidade aberta por 24 horas sem novas crises de angioedema, recebendo alta no dia seguinte.

\section{Discussão}

$\mathrm{O}$ angioedema hereditário é uma doença genética de padrão autossômico dominante. Em sua forma mais comum, os pacientes com a patologia apresentam deficiência quantitativa (80\%-85\%) e/ou qualitativa $(15 \%-20 \%)$ da enzima inibidora de $\mathrm{C} 1$-esterase (C1-INH). Um terceiro tipo descrito seriam os pacientes com níveis normais de C1-INH, porém com mutação do gene do fator XII da coagulação ou de origem desconhecida. ${ }^{1,3}$

A C1-INH é responsável por várias vias inflamatórias, coagulação e do complemento (Figura 3). Na via inflamatória mediada pela 
Figura 1. Edema em lábio inferior.

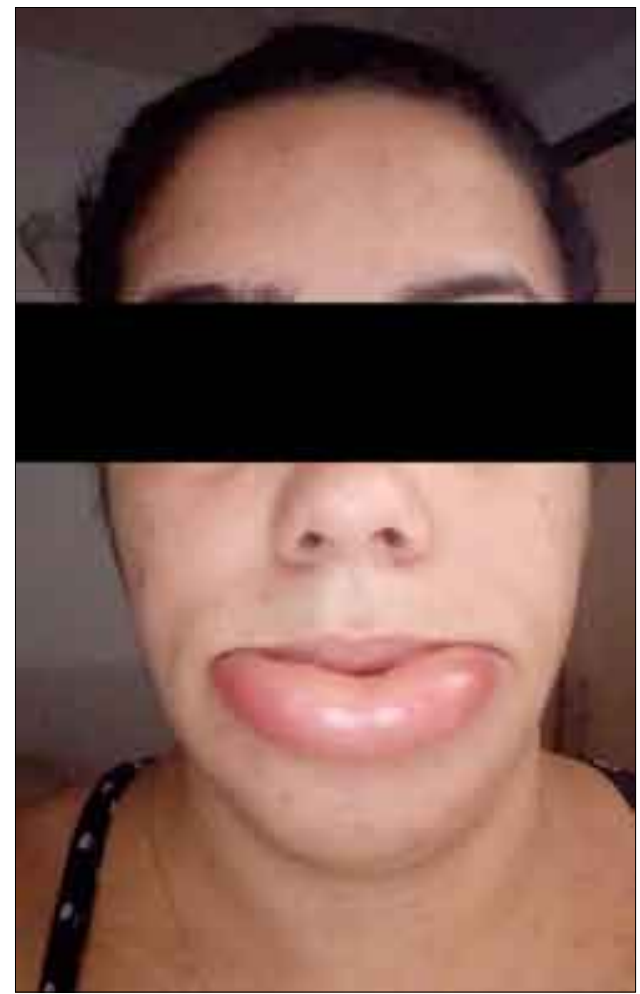

Fonte: Foto autorizada (arquivo pessoal da paciente).
Figura 2. Edema em mão após o procedimento.

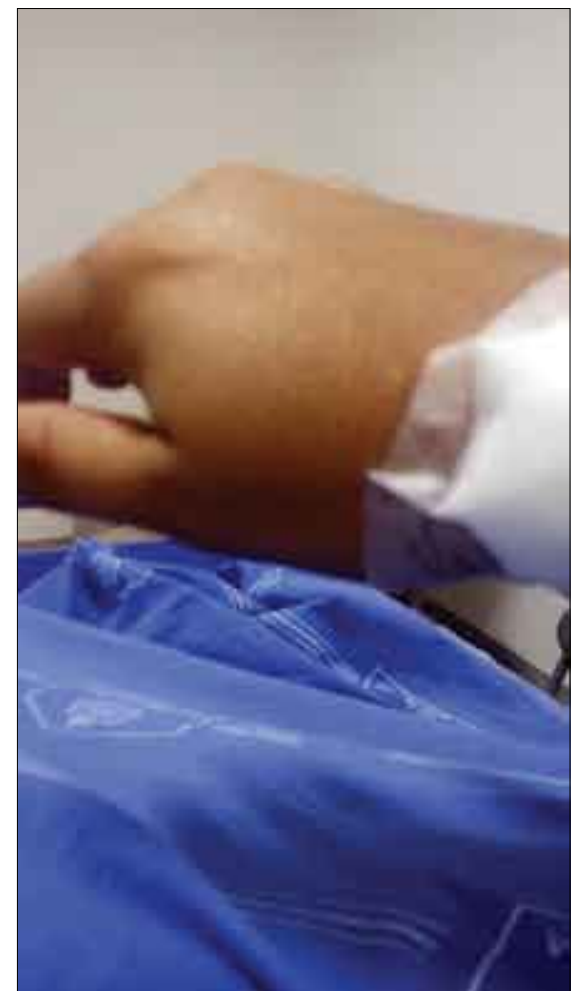

Fonte: Foto autorizada (arquivo pessoal da paciente). calicreína, a C1-INH controla a conversão de cininogênio de alto peso molecular em bradicinina, regulando a vasodilatação e aumento da permeabilidade vascular. Desta forma, pacientes com deficiência da C1INH, na presença de fatores desencadeantes como estresse, pequenos traumas, infecções, alterações hormonais (gravidez, menstruação, contraceptivos orais com estrógenos), uso de inibidores da enzima conversora de angiotensina (IECA) e procedimentos cirúrgicos, podem apresentar sintomas de angioedema em pele e mucosas.

$\mathrm{O} A E H$ caracteriza-se por ser um quadro de edema localizado, assimétrico, nãoinflamatório, não-pruriginoso e auto limitado (melhora espontânea após 48-72 horas) que acomete principalmente abdome, laringe, face, língua, extremidades e genitália. O edema laríngeo, apesar de ser menos frequente $(<1 \%$ dos episódios), é a complicação mais grave e causa da maior mortalidade relacionada ao $\mathrm{AEH}(25 \%$ a $40 \%){ }^{1,3}$ As crises têm como principal diagnóstico diferencial as reações anafiláticas (angioedema histaminérgico), porém a presença de urticária pruriginosa característica destas últimas torna o diagnóstico de AEH menos provável. ${ }^{1,4}$

No contexto da anestesiologia, o conhecimento prévio da patologia tem importância no manejo adequado de possíveis complicações, ${ }^{5}$ como edema de vias aéreas, visto que,por se tratar de um processo inflamatório mediado via bradicinina, o uso de anti-histamínicos, corticoides e epinefrina não são efetivos no tratamento. ${ }^{3} \mathrm{O}$ manejo adequado do AEH é feito com tratamento profilático (curto e longo prazo) e das crises agudas de angioedema.

A profilaxia a longo prazo tem como propósito diminuir a frequência e gravidade das crises. A profilaxia a curto prazo, por 
Figura 3. Ativação do sistema calicreína-cininas no angioedema hereditário.

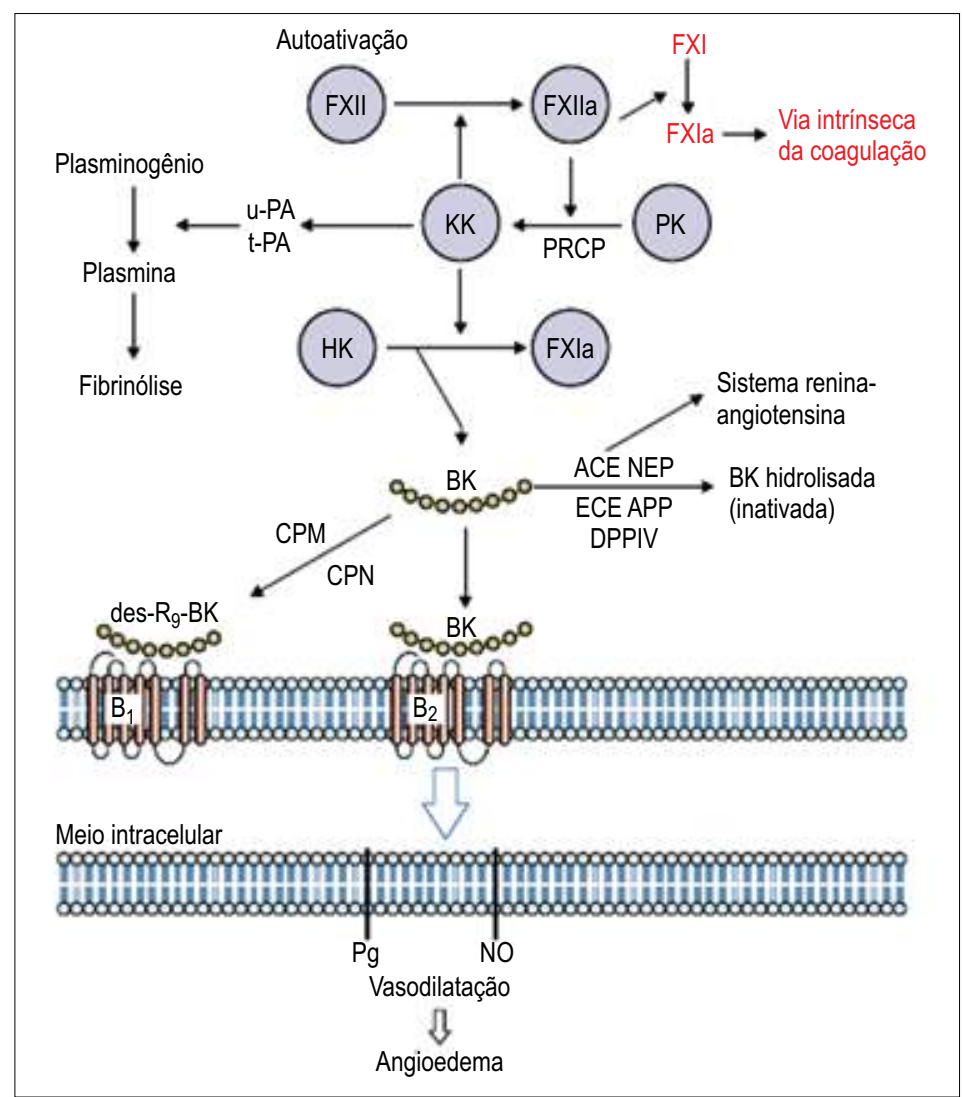

$\mathrm{ACE}=$ enzima conversora de angiotensina, $\mathrm{APP}=$ aminopeptidase $\mathrm{P}, \mathrm{DPPIV}=$ dipeptidil peptidase IV, des-R9-BK $=\mathrm{BK}$ sem a arginina na posição 9, ECE = enzima conversora de endotelina, FXIIa = FXII ativado, FXI = fator XI da coagulação, $\mathrm{FXIa}=\mathrm{FXI}$ ativado, $\mathrm{HK}=$ cininogênio de alta massa muscular, $\mathrm{HKa}=\mathrm{HK}$ livre de $\mathrm{BK}, \mathrm{KK}=$ calicreína plasmática, NEP $=$ endopeptidase neutra, $\mathrm{u}-\mathrm{PA}=$ ativador de plasminogênio tipo uroquinase, $\mathrm{t}-\mathrm{PA}=$ ativador de plasminogênio tecidual, PRCP = prolil-carboxi-peptidase, $\mathrm{PK}=$ pró-calicreína plasmática. (Fonte: Diretrizes Brasileiras para o Diagnóstico e Tratamento do Angioedema Hereditário - 2017). ${ }^{1}$

sua vez, tem o objetivo de prevenir crises de angioedema durante procedimentos com risco de comprometimento das vias aéreas, como tratamento dentário invasivo (extração dentária), amigdalectomia, cirurgia facial, endoscopia, broncoscopia ou procedimentos que necessitem de intubação traqueal. ${ }^{1,4,5}$

Além do risco de complicações relacionadas às vias aéreas, a profilaxia a curto prazo é feita considerando-se a disponibilidade de tratamento para a crise aguda. Podem ser utilizados andrógenos atenuados, agentes antifibrinolíticos, concentrado de C1-INH derivado de plasma (pdC1-INH) e/ou plasma fresco congelado (Quadro 1)., ${ }^{1,4}$
No manejo da crise aguda deve-se manter a permeabilidade da via aérea, oxigenação e analgesia adequadas. Nos casos leves é indicado o uso de ácido tranexâmico (antifibrinolítico) e, em casos mais graves, o tratamento de escolha inclui a reposição do concentrado de $\mathrm{C} 1-\mathrm{INH}$ derivado de plasma, bloqueio dos efeitos da bradicinina - com icatibanto (antagonista do receptor de bradicinina) ou ecallantide (inibidor de calicreína) - e plasma fresco (Quadro 2). Este último deve ser utilizado apenas na indisponibilidade de outras opções terapêuticas devido aos seus inúmeros efeitos colaterais e porque, apesar de conter o $\mathrm{C} 1$-INH que promove 
Quadro 1. Profilaxia de curto prazo para crises de angioedema hereditário.

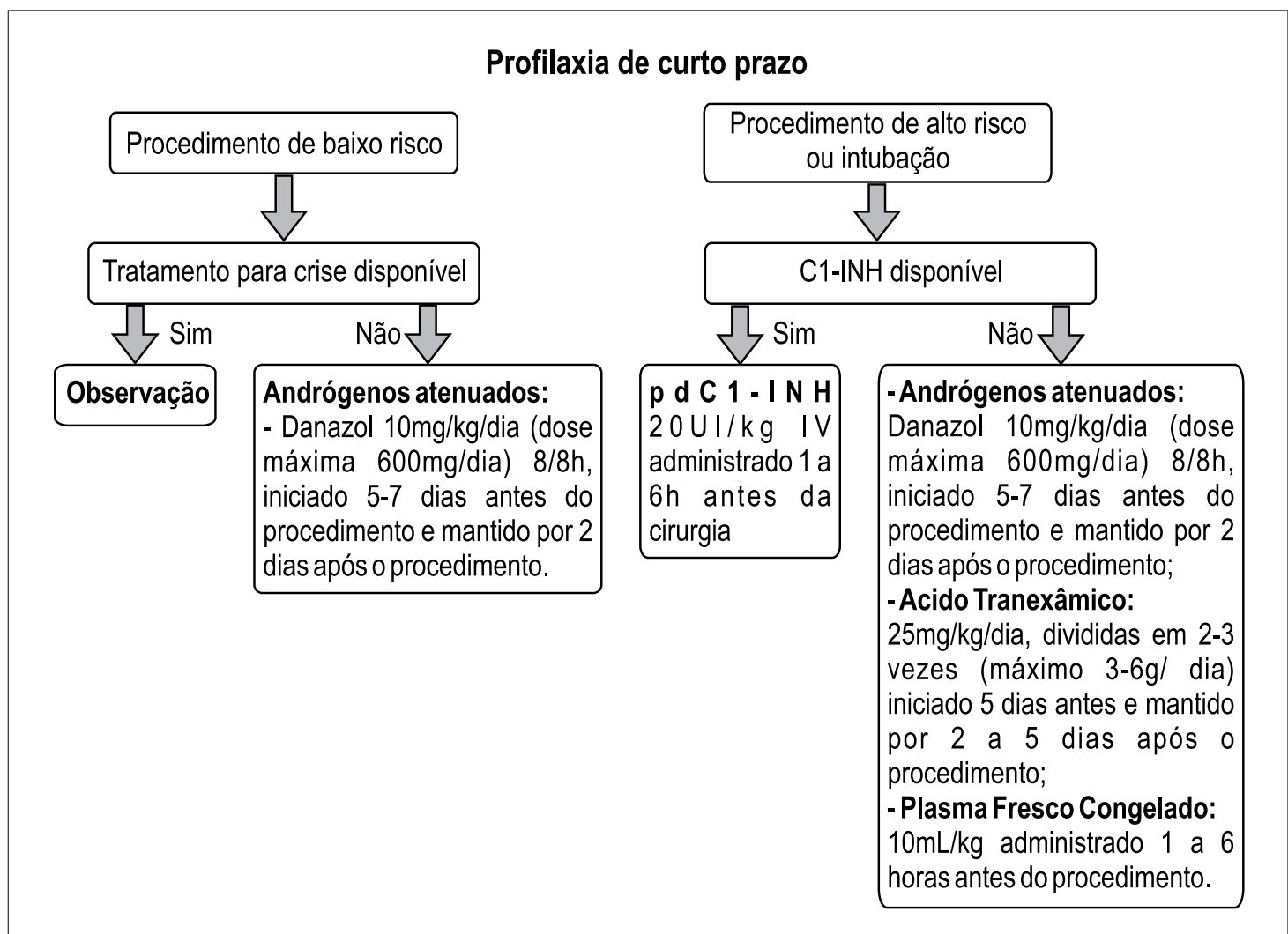

Quadro 2. Manejo da crise aguda.

\section{Tratamento da Crise Aguda}

Manutenção da permeabilidade das vias aéreas, oxigenação adequada, analgesia

Reposição do C1-INH

- Concentrado de $\mathrm{C} 1-\mathrm{INH}$

e C1-INH recombinante

- Plasma fresco congelado

\section{Bloqueio dos efeitos da Bradicinina:}

- Icatibanto (antagonista do receptor de bradicinina do tipo II)

- Ecallantide (inibidor de calicreína)

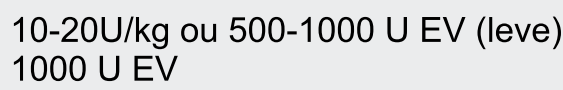

$10 \mathrm{ml} / \mathrm{kg}$

$30 \mathrm{mg} /$ dose SC e repetir de $6 / 6 \mathrm{~h}$ se a crise persistir (máximo 90mg/dia)

$30 \mathrm{mg} /$ dose SC e repetir segunda dose se a crise persistir

*Agentes antifibrinolíticos (ácido tranexâmico $1 \mathrm{~g}$ a cada 4 horas por via oral) podem ser utilizados em crises leves. 
a melhora da crise, também possui componentes que teoricamente poderiam retroalimentar a cascata de formação da bradicinina. Entretanto, por sua maior disponibilidade, coloca-se como uma opção terapêutica aos casos agudos na ausência do tratamento específico. ${ }^{1,4}$

$\mathrm{O}$ angioedema hereditário é uma condição clínica rara, porém de grande morbi-mortalidade caso não seja diagnosticado e tratado da maneira correta. Os pacientes submetidos à anestesia, mesmo que para exames ou cirurgias de pequeno porte e a nível ambulatorial, devem ser observados quanto ao aparecimento de edema $\mathrm{e}$ comprometimento das vias aéreas após o estresse cirúrgico. A identificação precoce de possíveis complicações e a instituição da terapêutica adequada são essenciais para garantir um melhor prognóstico para estes pacientes.

\section{Referências}

1. Giavina-Bianchi P, et al. Diretrizes brasileiras para o diagnóstico e tratamento do angioedema hereditário2017. Arq Asma Alerg Imunol. 2017;1(1):23-48.

2. Phelan PD, Robertson CF, Olinsky A. Pathways for bradykinin formation and inflammatory disease. Journal of Allergy and Clinical Immunology 2002;109(2):189-94.

3. Machado AF, Thomaz JE, Neto HJC, Riedi CA, Rosário NA. Angioedema hereditário: uma atualização. Rev Med UFPR. 2015;2(1):33-8.

4. Barbara DW, et al. Perioperative angioedema: background, diagnosis, and management. Journal of Clinical Anesthesia 2013;25(4):335-43.

5. MacBeth LS, et al. Perioperative course in patients with hereditary or acquired angioedema. Journal of Clinical Anesthesia 2016;34:385-91.

6. Savio KH, Callum J, Alam A. C1-esterase inhibitor for short-term prophylaxis in a patient with hereditary angioedema with normal $\mathrm{C} 1$ inhibitor function. Journal of Clinical Anesthesia 2016;35:488-91. 